\title{
Erratum: Analytic model of the energy spectrum of a graphene quantum dot in a perpendicular magnetic field [Phys. Rev. B 78, 195427 (2008)]
}

\author{
S. Schnez, Klaus Ensslin, M. Sigrist, and T. Ihn \\ (Received 5 December 2016; published 10 January 2017)
}

DOI: 10.1103/PhysRevB.95.039901

The use of the parameter $m$ in Eq. (11) of our publication is incorrect. Rather, it should read

$$
E= \pm v_{F} \sqrt{2 e \hbar B(m+1+p)}
$$

where $m$ is the previously defined quantum number and $p$ is an integer with $p>-(m+1)$. This follows from the fact that Eq. (6) in our publication can be simplified to $[\Gamma(\alpha) \Gamma(-\alpha)]^{-1}=0$ in the limit $R / l_{B} \rightarrow \infty$ with $\alpha:=k^{2} l_{B}^{2} / 2-m-1$. This is fulfilled for $\alpha= \pm p$ and $p$ being an integer. The later restriction of $p>-(m+)$ is then required to make the radicand non-negative.

The mathematical derivation based on our ansatz as described in our original publication is correct. 